OSTRIKER, J. P., 1971, Annual Reviews of Astronomy and Astrophysics, 9, 353.

OSTRIKER, J. P., and HESSER, J. E., 1968, Astrophys. J. 153, L151 (OH).

OSTRIKER, J. P., and TASSOUL, J. L., 1968, Nature 219, 577.

ROSE, W. K., 1967, Astrophys. J. 150, 193.

SINGLETON, R. C., 1967, IEEE Trans. in Audio and Electroacousties 15, 91.

SMAK, J., 1967, Acta Astronomica 17, 255.

VILA, S. C., 1970, Astrophys. J. 162, 971.

VILA, S. C., 1971, Astrophys. J. 163, 543.

WALKER, M. F., 1954, Pub. Astron. Soc. Pacific 66, 230.

WALKER, M. F., 1967, in Non-Stable Stars (ed. by G. Herbig), Cambridge University Press, Cambridge, p. 46

WARNER, B., and NATHER, R. E., 1970, Mon. Not. Roy. Ast. Soc. 147, 21.

WARNER, B., VAN CITTERS, G. W., and NATHER, R. E., 1970, Nature, 226, 67.

\title{
Variable X-Ray Sources
}

\author{
EDWIN M. KELLOGG (Cambridge, USA)
}

\section{Introduction}

There are at least five types of variable galactic X-ray sources (i. e. "stars") known:

\author{
Pulsar, as NP0532 \\ Transient Sources as Cen XR-2 \\ Quasi-periodic rapid variables, as $\mathrm{Cyg} \mathrm{X}-1$ \\ Periodic rapid variables, as Cen $\mathrm{X}-3$ \\ Aperiodic variables, as Sco X-1.
}

In this paper, $I$ will not discuss the pulsar or the transient sources, as we at AS \& $E$ have not made any new observations of these sources yet with the UHURU satellite. The paper by RAPPAPORT (1970) reviews the observations of NP0532 to that date. Since then, HILLIER, JACKSON, MURRAY, REDFERN and SALE (1971) have observed it above $0.6 \mathrm{MeV}$. The transient sources Cen XR-2 which appeared in 1967 and Cen XR-4 which appeared in 1969 have not reappeared in the UHURU data, although the previous position measurement accuracy for these sources was not sufficient to allow us to rule out a possible weak remaining source.

The main emphasis in the following will be to describe the behavior of the quasiperiodic rapid variable $\mathrm{C}_{y g} \mathrm{X}-1$, the periodic variable $\mathrm{Cen} \mathrm{X}-3$, and the aperiodic variable Sco X-1. These objects are not well understood. The bulk of the $\mathrm{X}$-ray data on them were accumulated in the past six months, so models of them have not had much time to be formulated. However, I will try to summarize briefly the current trends of thought on the nature of these sources.

\section{Cyg X-1: A Quasi-Perlodic Rapid Variable}

Intensive studies have been done on $\mathrm{Cyg} X-1$ at $\mathrm{AS} \& \mathrm{E}$, since we recently discovered that it has rapid fluctuations in intensity (see ODA et al., 1971). The UHURU satellite was pointed to a position where $\mathrm{Cyg}_{\mathrm{g}} \mathrm{X}-1$ was in the band visible to the $\mathrm{X}$-ray detectors, and the spin rate was slowed down to one revolution per hour to allow continuous observations of the source up to $100 \mathrm{sec}$. One such sample of data is shown in Figure 1. The count rate from the source is estimated by onboard accumulation of counts for $0.096 \mathrm{sec}$. Figure 1 (a) shows a plot of these sequential data samples with the same $0.096 \mathrm{sec}$. time resolution as received from the satellite. Parts (b), (c) and (d) are plots with coarser time resolution obtained by summing over several data points at a time, so that the accumulation time is $0.48,4.8$ and $14.4 \mathrm{sec}$. respectively. Significant fluctuations in the intensity of the source are present in each case. A large fraction of the total X-ray power emitted by Cyg X-1 is fluctuating in this way. 

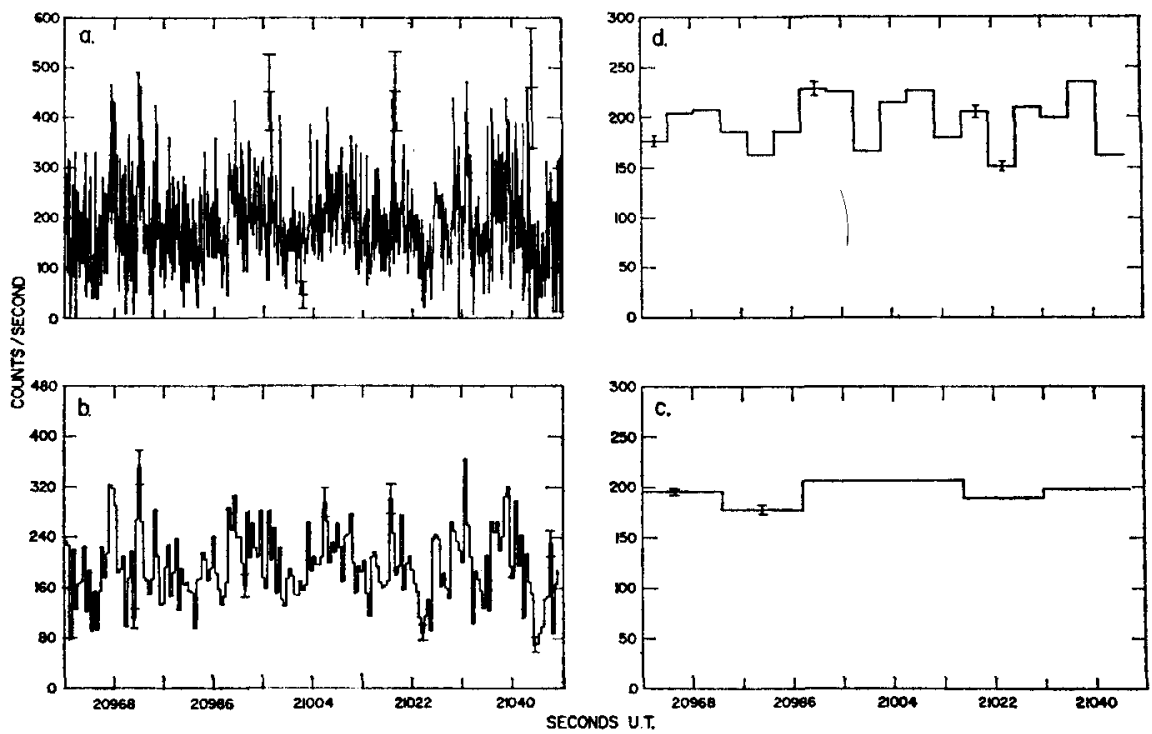

Fig. 1: Observation of Cygnus X-1 on June 10, 1971, made using UHURU. Data have been corrected for triangular collimator response. a) Data with 0.096 second resolution; b), c) and d) Data summed over intervals of $0.48 \mathrm{sec} ., 4.8 \mathrm{sec}$. and $14.4 \mathrm{sec}$. Typical one-sigma error bars are shown.

The next question is whether these fluctuations are periodic. The fourier spectrum of these fluctuations is shown in Figure 2. Significance of a peak at the $90 \%$ confidence level is indicated by the dashed line. Two periods are indicated in the present data, 2.28 and $4.56 \mathrm{sec}$. However, when ten other such $100 \mathrm{sec}$. samples of data were analyzed, there was no reappearance of either period. In each case, however, some period in the range 0.1 to $20 \mathrm{sec}$. was found. Further studies (see SCHREIER, GURSKY, KELLOGG and TANANBAUM, and GIACCONI, 1971) show that these periodicities have a lifetime of no more than a few tens of seconds. We conclude that $\mathrm{Cyg} \mathrm{X-1}$ is quasi-periodic in the range 0.05 to $20 \mathrm{sec}$ We have observed variations on a time scale of minutes to hours as well, of order a factor of two in change of intensity.

No consistent model of the nature of $\mathrm{Cyg} X-1$ has been proposed yet. Such a model should explain the optical radiation from the direction of Cyg X-1 (or lack thereof), (see KRISTIAN, BRUCATO, VISVANATHAN, LANNING and SANDAGE, 1971) as well as the radio emission observed by HJELLMING and WADE, 1971, BRAES and MILEY, 1971, and the hard X-rays up to $100 \mathrm{keV}$ seen by many observers. The only definite conclusion we can make at present is that the $\mathrm{X}$-ray emitting region is a few light sec. in size, and some of the fast variations originate in a region as small as several earth radii in size.

\section{Cen X-3: A Periodic Variable}

After we discovered in the UHURU data that $\mathrm{C}_{\mathrm{yg}} \mathrm{X}-1$ was varying so strangely, we began to examine data from the other strong sources for pulsations. It was almost immediately obvious that Cen X-3 was varying in intensity periodically. The clearest illustration of this effect is given in Figure 3. These data are from a 140 second scan of Cen X-3. The overall triangular envelope is the collimator response of the $\mathrm{X}$-ray detector system as it scans over the source. A source of constant intensity would give a count rate versus time histogram 
which reproduced the triangle exactly, except for small statistical fluctuations from sample to sample. What we see is a histogram showing the source intensity to be modulated with a period of about $4.8 \mathrm{sec}$. The form of the modulation as determined by Fourier analysis shows a dominant fundamental frequency component at about $4.8 \mathrm{sec}$. and a strong first harmonic at about $2.4 \mathrm{sec}$, about $30 \%$ as strong as the fundamental. No higher frequency component shows up. The two frequencies combine to give a pulse shape characterized by a fast rise and a slower decay, or shoulder which might be due to an interpulse.

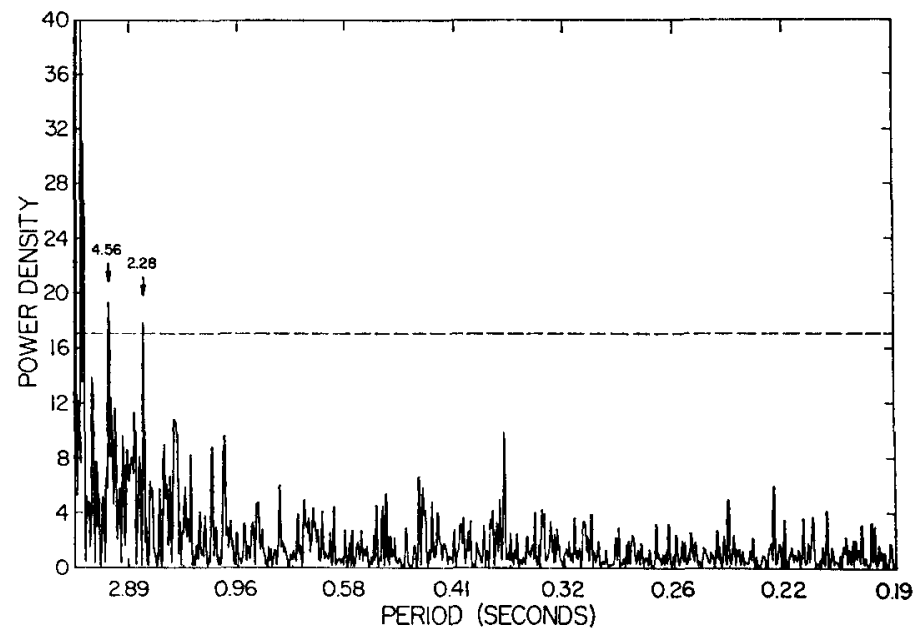

Fig. 2: Power density spectrum from Fourier analysis of a 110 second observation of Cygnus $\mathrm{X}-1$ on June 11,1971 , with 0.096 second time resulution. Power density is computed from the Fourier amplitude squared normalized by he total number of counts. The probability that any peak in the power spectrum of an equivalent interval of normally distributed data will exceed the level indivated by the dashed line is $10 \%$. Periods above about 20 seconds are due to triangular response of collimator.

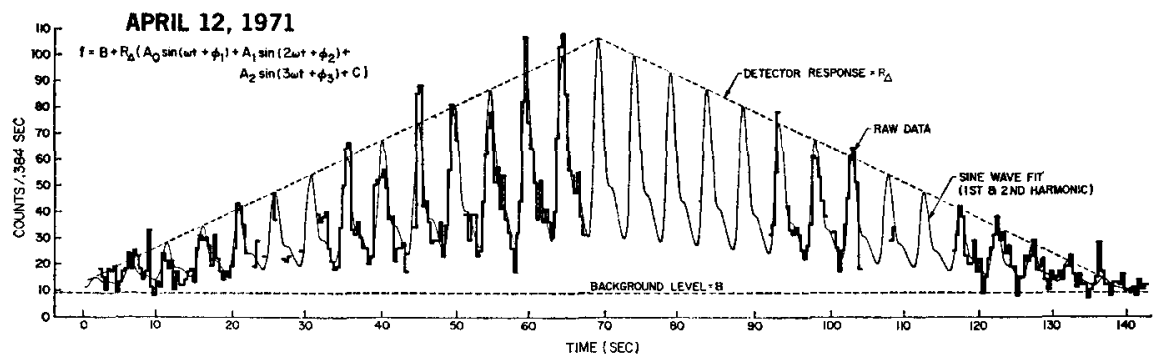

Fig. 3: UHURU data from Cen X-3. Histogram shows counts accumulated in $0.38 \mathrm{sec}$. intervals in the $2-6 \mathrm{keV}$ energy range. Missing portions of the histogram are due to gaps in the transmission of quick-look data from the satellite to our laboratory, not due to characteristics of Cen X-3. The dashed line labelled detector response in the profile that would be followed by the data if Cen X-3 were not varying. The solid line curve is the best fit of a function $f$ (defined on the legend to the figure) to the data. 


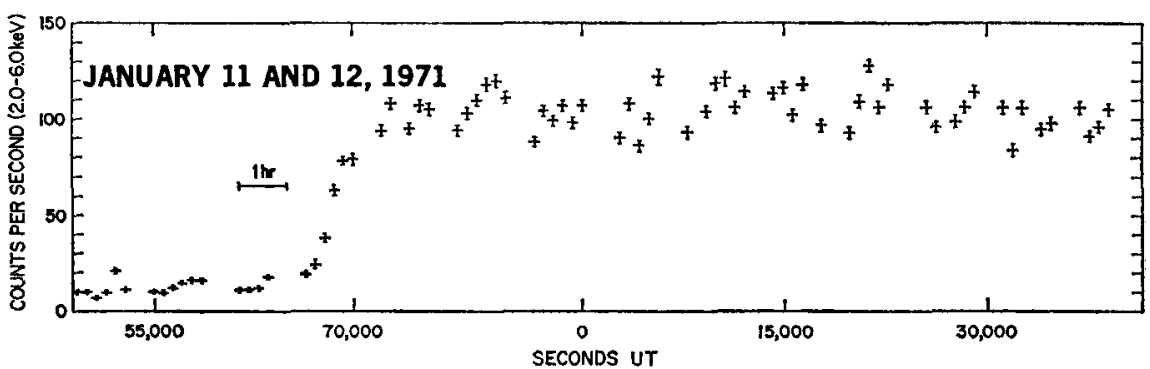

Fig. 4: Average count rate from Cen X-3 in the 2 to $6 \mathrm{keV}$ energy range as a function of time observed on January 11 and 12, 1971. Error bars reflect only counting statistics. Missing data are due to occultation of the source by the earth. Data are not corrected for aspect, so the apparent variability after $70000 \mathrm{sec}$. January 11 may not be real.

Figure 4 shows the intensity versus time of $\mathrm{Cen} X-3$ over about one day; with a dramatic rise by a factor of ten occurring. We have seen Cen $\mathrm{X}-3$ in both states on various occasions. When it is weak, it is difficult to say whether it is pulsing but we believe it is still pulsing with a 4.87 sec. period.

This object was only discovered a few months ago; any explanation of its nature is still incomplete. However, at the present time it is believed that a pulsating white dwarf could explain its X-ray behavior. As the surface of the star pulsates, an outgoing shock wave develops in its atmosphere, which eventually heats an outer layer to a sufficient temperature for X-ray emission. The cooling time is expected to be fast enough for such a layer that it would cool completely between pulses, and the pulsing would not be smeared out (see BLUMENTHAL et al., 1971).

\section{Aperiodic Variables: Sco X-1}

Sco X-1 is the brightest X-ray source, and emits 100 times more power in X-rays than in visible light. It has been identified with a blue star which varies from $12.4-13.2 \mathrm{mg}$. It is also identified with a highly variable radio source with complex structure.

Simultaneous observations of Sco X-1 were done in March 1971 by UHURU, Westerbork. NRAO, Leiden Southern Station, Cerro Tololo, Kitt Peak and McDonald Observatory. Some of the X-ray and optical data are shown in Figure 5.

The $\mathrm{X}$-ray data are the thin vertical lines which represent intensity measurements $\pm \sigma$. The optical data, covering about 2 hours each day, are from Cerro Tololo, taken by HALE BRADT and WILLIAM KUNKEL, and are indicated by Xes. The X-ray data reveal that Sco X-1 behaves in soft $\mathrm{X}$ rays much as it is well known to do in visible light. That is, it has a quiet state $(\sim 8000$ counts $/ \mathrm{sec}$.) and a bright, flaring state $(10,000-17,000$ counts $/ \mathrm{sec}$.$) .$ There is some evidence for a correlation between the X-ray and optical intensity; especially note the sudden change to the bright flaring state on March 26 . However, we must fill in more optical data which are still being reduced.

There was no obvious correlation between X-ray or optical intensity and $3.7,11.7$ or $21 \mathrm{~cm}$ radio intensity during this period.

The emission in X-rays and optical from Sco X-1 can be explained as due to a thin hot plasma with effective temperature varying from $40-160$ million degrees Kelvin. The plasma exciting mechanism is not yet determined, although it becomes optically thick in the infrared. Also, no periodicity has yet been found in its light curve, either in X-rays or visible light or radio.

\section{Adknowledgements}

The work reported here was done by several of us at AS \& E. The others are R. GIACCONI, H. GURSKY, S. MURRAY, E. SCHREIER, H. TANANBAUM, W. TUCKER and G. BLUMENTHAL. I also acknowledge with pleasure the dedicated and enthusiastic participation of HALE 
BRADT, WILLIAM KUNKEL and W. A. HILTNER in the cooperative effort which helped to make simultaneous $\mathrm{X}$-ray and optical observations possible. This work was supported by the U.S. National Aeronautics and Space Administration under Contract NAS 5-11422.

References:

BLUMENTHAL, G., CAVALIERE, A., ROSE, W., and TUCKER, W., 1971, Ap. J., to be published. HILLIER, JACKSON, MURRAY, REDFERN and SALE, 1970, Ap. J. 162, L177.

H JELLMING, R., and WADE, C., 1971, Ap. J. 168, L21.

KRISTIAN, J., BRUCATO, R., VISVANATHAN, J., LANNING, H., and SANDAGE, A., 1971, Ap. J. 168, L91.

BRAES, L., and MILEY, G., 1971, Nature 232, 246.

ODA, M., GORENSTEIN, P., GURSKY, H., KELLOGG, E., SCHREIER, E., TANANBAUM, H., and GIACCONI, R., 1971, Ap. J. 166, L1.

RAPPAPORT, S 1970. IAU Symposium No. 46, The Crab Nebula, Jodrell Bank.

SCHREIER, E., GURSKY, H., KELLOGG, E., TANANBAUM, H., and GIACCONI, R., 1971, Ap. J. Letters, to be published.
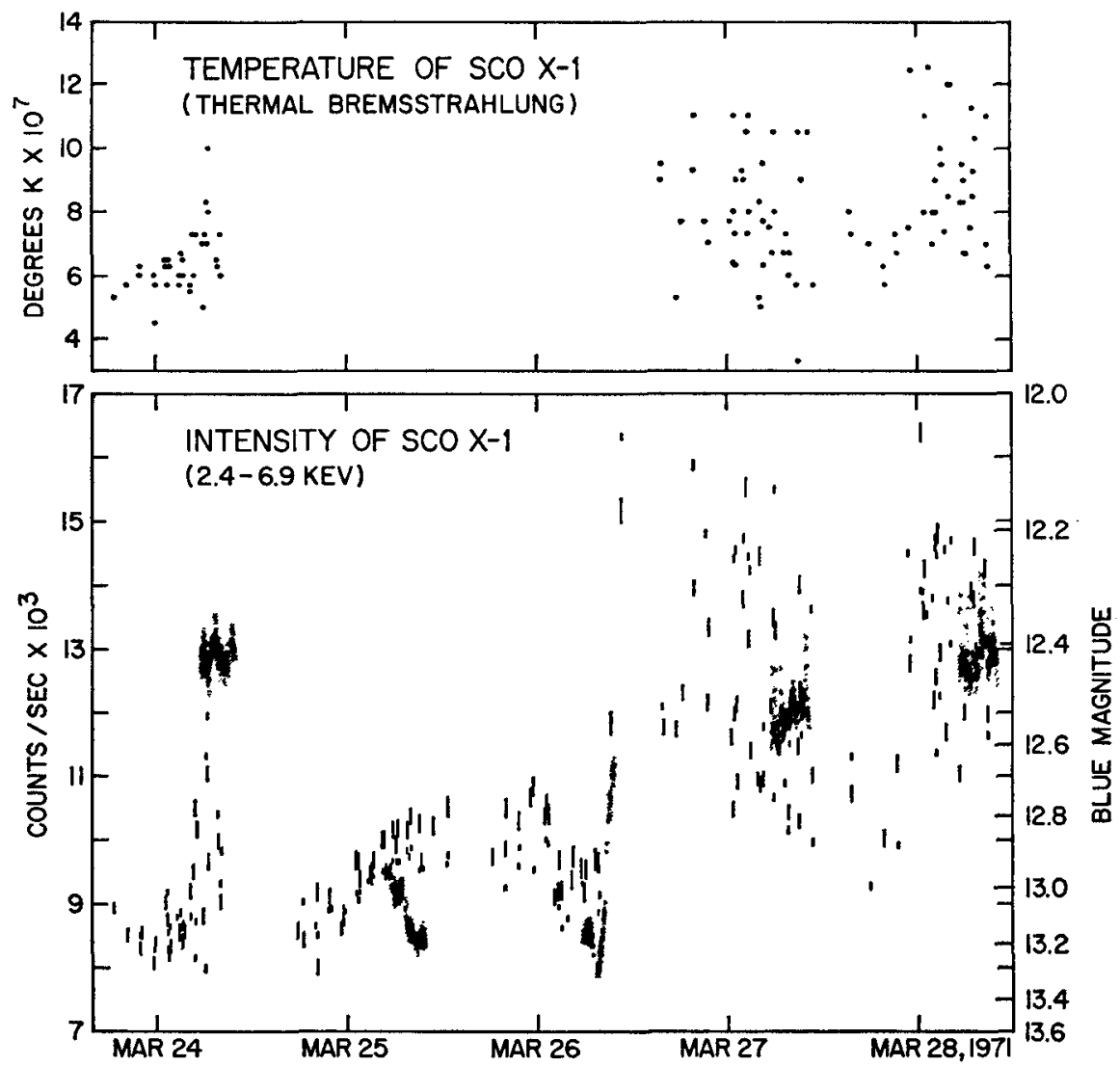

Fig. 5: X-ray and optical intensity vs. time for Sco X-1. Vertical lines are X-ray sightings, $\pm 1 \sigma$. Xes are blue magnitude. Upper graph shows $X$-ray "temperature" vs. time. The temperature parameter is obtained by fitting the $\mathrm{X}$-ray pulse height spectrum obtained by UHURU on each sighting, to an assumed thermal bremsstrahlung energy spectrum of exponential form. 